\title{
A Brief Overview and Experimental Researches of Novel PL-log-MAP Turbo Decoding Algorithm
}

\author{
A.N. Romanyuk, Yu.Yu. Ivanov \\ Faculty of Automatics, Electronics and Computer Systems \\ Vinnytsia National Technical University, Vinnytsia, Ukraine \\ e-mail: ran12345@mail.ru, YuraII@yandex.ru \\ O.V. Stukach \\ Department of Control Systems and Mechatronics \\ National Research Tomsk Polytechnic University, Tomsk, Russia
}

\begin{abstract}
In the paper, we will discuss the powerfull class of channel codes referred to as turbo codes. We commence with a brief discussion for MAP and log-MAP decoding algorithms. Then we work with proposed turbo decoding method, which is called as PL-log-MAP. Some numerical results and research experiments, such as simulation for bit error rate estimation and images transmission have been presented. It is shown that PL-logMAP algorithm performance is almost the same as for original log-MAP. So, this algorithm can be used in digital communication systems.
\end{abstract}

Keywords: turbo code, MAP, log-MAP, PL-log-MAP, correction function, approximation algorithms, piecewise linear techniques, numerical results, simulation, experiments.

\section{INTRODUCTION}

One of the most interesting and most promising direction in the development of an error correction theory is data protection, which is based on cascaded parallel code structures (often with convolutional component codes) or turbo codes. The term "turbo" and important mathematical apparatus for the turbo code was first proposed in the fundamental work [1]. It is shown that at the high bit error rate $\left(\mathrm{BER}=10^{-5}-10^{-7}\right.$ from 0 to $3 \mathrm{~dB}$ ) the best of the considered codes is turbo code (power gain of about 7-9 dB), what is very close to Shannon limit [3]. Thus, turbo code is the most effective method for information transmission in channels with low energy consumption. The results of original work [1] gave a powerful impetus to scientists to perform detailed research in the turbo decoding field with using of complex computational algorithms with high degree of error correction [2]. High turbo code efficiency is caused by special iterative decoding algorithms SOVA, Bi-SOVA, MAP or BCJR, log-MAP and other modifications [4-9, 12, 14]. All turbo decoding algorithms can be interpreted as the implementation of techniques known as message passing algorithm or belief propagation algorithm. To describe the strategy, that is embodied in an iterative decoding of turbo codes, a German scientist J. Hagenauer introduced the concept of "turbo"principle: supply output data back to the entrance, which is similar with turbocharger engine; concept of probability iterative decoders with soft input and soft output; parallel working of elementary coders $[4,10]$.

Turbo codes can be implemented in software, hardware or mixed (software and hardware) manner. Often turbo codes implemented in mixed manner on digital signal processors. Detailed researches of turbo decoding complexity is given in [13] as well as comparative analysis for implementation complexity of turbo decoding algorithms.

Turbo codes with probabilistic decoding algorithms are used for high performance data transmission in almost any communication system: television (DVB-RCS, DVB-RCT, DVB-SSP), telemetry, wireless local area networks (WiMAX), SDR systems (software defined radio), mobile (3G, $4 \mathrm{G}$, Inmarsat) and space (CCSDS) communication [11].

Since there is no adequate mathematical tools that would calculate the practical error correction characteristics for turbo code, then computer simulation with Monte-Carlo method is used. So the purpose of this work is to conduct simulation for data transmission process in channels with low energy consumption for research an important experimental dependencies for the turbo code using developed by us in [15] piecewise-linear log-MAP decoding algorithm (PL-log-MAP). This algorithm helped to reduce computational complexity without significantly reducing of decoding quality.

The paper is structured as follows. Section II is connected with the basic of iterative turbo decoding with MAP and logMAP algorithms and their modifications. In Section III we compare some numerical results of PL-log-MAP decoding algorithm with other existing algorithms. At final we simulate the work of digital communication system with turbo codec and using PL-log-MAP to decode images in MatLab. The paper is finally concluded in Section IV.

\section{DESCRIPTION OF TURBO CODE DECODING AlgORITHMS}

In this section, we will present MAP algorithm not in details, but the main features of the algorithm will be stated. 
An overview of the log-MAP algorithm will be presented. Detailed description is given in $[4-9,11,15,16]$.

The BCJR algorithm for convolutional codes was first presented in the fundamental work [12]. Nowadays in modern coding theory this algorithm has several names: MAP (maximum a posteriori probability), APP (for a posteriori probability), BCJR (the first letters of the authors surnames), FBA (forward-backward algorithm), SPA (sum-product algorithm). MAP or BCJR decoders show better energy efficiency, but they are more difficult to implement, than SOVA decoders [16].

The basis of the mathematical apparatus for turbo decoding is some computational procedure for assessing the solutions reliability, which is called logarithm of the likelihood ratio or LLR.

$$
\left.\operatorname{LLR}_{M A P}\left(d_{k}\right)=\ln \frac{p\left(d_{k}=+1 \mid x\right)}{p\left(d_{k}=-1 \mid x\right)}=\ln \frac{\sum_{d_{k}=+1}^{\left(s^{\prime}, s\right)} p\left(s^{\prime}, s, x\right)}{\sum_{d_{k}=-1}^{\left.S^{\prime}, S\right)} p\left(s^{\prime}, s, x\right)}\right)_{H_{2}}^{H_{1}} 0,
$$

where $d_{k}$ is information symbol; $s$, and $s$ is past and present states at trellis; $x_{k}$ is received sequence; $H_{1}, H_{2}$ is the hypotheses and if $\operatorname{LLR}_{M A P}\left(d_{k}\right)>0$ or $H_{1}$ is true, the hard binary decision will be 1 , in otherwise $-0, \ln ($.$) is natural$ logarithm.

A posteriori soft decisions for MAP algorithm can be represented as the sum of three measurements or $L L R \mathrm{~s}-$ channel, a priori (intrinsic) and external, as reflected in the following equation

$$
\begin{array}{r}
L L R_{M A P}\left(d_{k}\right)=\ln \frac{\sum_{d_{k}=+1}^{\left(s^{\prime}, s\right)} \alpha_{k-1}\left(s^{\prime}\right) \cdot \gamma_{k}\left(s^{\prime}, s\right) \cdot \beta_{k}(s)}{\sum_{d_{k}=-1}^{\left(s^{\prime} s\right)} \alpha_{k-1}\left(s^{\prime}\right) \cdot \gamma_{k}\left(s^{\prime}, s\right) \cdot \beta_{k}(s)}= \\
=L L R_{c h} \cdot x_{k}+L L R_{a p r}\left(d_{k}\right)+L L R_{e x t}\left(d_{k}\right),
\end{array}
$$

where $\alpha_{k}(s)=\sum_{\left(s^{\prime}, s\right)} \gamma_{k}\left(s^{\prime}, s\right) \cdot \alpha_{k-1}\left(s^{\prime}\right)$ is forward path metric on the trellis for recursive systematic convolutional $(\mathrm{RSC})$ code; $\beta_{k-1}\left(s^{\prime}\right)=\sum_{\left(s^{\prime}, s\right)} \gamma_{k}\left(s^{\prime}, s\right) \cdot \beta_{k}(s)$ is backward path metric on the trellis for RSC code; $\gamma_{k}\left(s^{\prime}, s\right)$ is transit or rib metric.

Efficient modification is log-MAP algorithm. It works in logarithmic area (natural logarithm of relevant MAP metrics is calculated and some simplifications is performed), has a lower computational complexity and achieves performance of MAP turbo decoding algorithm. For example, the forward path metric is given as

$$
\begin{aligned}
\alpha_{k}^{L M}\left(s^{\prime}\right) & =\ln \alpha_{k}\left(s^{\prime}\right)=\ln \left(\sum_{\left(s^{\prime}, s\right)} \gamma_{k}\left(s^{\prime}, s\right) \cdot \alpha_{k-1}\left(s^{\prime}\right)\right)= \\
& =\ln \left(\sum_{\left(s^{\prime}, s\right)} \exp \left(\ln \gamma_{k}\left(s^{\prime}, s\right)\right) \cdot \exp \left(\ln \alpha_{k-1}\left(s^{\prime}\right)\right)\right)=(3) \\
& =\ln \left(\sum_{\left(s^{\prime}, s\right)} \exp \left(\gamma_{k}^{L M}\left(s^{\prime}, s\right)+\alpha_{k-1}^{L M}\left(s^{\prime}\right)\right)\right) .
\end{aligned}
$$

Similarly, we present the backward path metric in the next equation

$$
\beta_{k-1}^{L M}\left(s^{\prime}\right)=\ln \beta_{k-1}(s)=\ln \left(\sum_{\left(s^{\prime}, s\right)} \exp \left(\gamma_{k}^{L M}\left(s^{\prime}, s\right)+\beta_{k}^{L M}(s)\right)\right) \text {. }
$$

The rib metric $\gamma_{i}^{(j)}\left(m^{\prime}, m\right)$ will have next form

$$
\begin{aligned}
& \gamma_{k}^{L M}\left(s^{\prime}, s\right)=\ln \gamma_{k}\left(s^{\prime}, s\right)= \\
& =\frac{1}{2} \cdot\left(d_{k} \cdot L L R_{\text {апр }}\left(d_{k}\right)+d_{k} \cdot L L R_{\text {кан }} \cdot x_{k}+\left(\sum_{v=2}^{n} d_{k, v} \cdot L L R_{\text {кан }} \cdot x_{k, v}\right)\right.
\end{aligned}
$$

Calculation of the logarithm of the exponential components sum is performed using Jacobian logarithm formula [7-9, 11, $15,16]$

$$
\begin{gathered}
\log (\exp (T)+\exp (Y))=\max (T, Y)+\log (1+\exp (-|T-Y|))= \\
=\max (T, Y)+f(|T-Y|)=\max (T, Y)+f_{\text {cor }}(z) .
\end{gathered}
$$

Note that in (6) can be neglected calculation of the correction function $f_{\text {cor }}$. In this case we lose of about $10 \%$ efficiency compared to the MAP method. Such suboptimal turbo decoding algorithm is called max-log-MAP [9].

To calculate a posteriori solutions for log-MAP decoding algorithm the logarithm of the $N$ exponential components sum is presented recursively. So the formula (2) can be written in the next form, where $L_{i}$ is the sum of forward, backward and rib metrics 


$$
\begin{aligned}
L L R_{L M}\left(d_{k}\right) & =\ln \frac{\sum_{d_{k}=+1}^{\left(s^{\prime}, s\right)} \exp \left(\alpha_{k-1}^{L M}\left(s^{\prime}\right)\right) \cdot \exp \left(\beta_{k}^{L M}(s)\right) \cdot \exp \left(\gamma_{k}^{L M}\left(s^{\prime}, s\right)\right)}{\sum_{d_{k}=-1}^{\left(s^{\prime}, s\right)} \exp \left(\alpha_{k-1}^{L M}\left(s^{\prime}\right)\right) \cdot \exp \left(\beta_{k}^{L M}(s)\right) \cdot \exp \left(\gamma_{k}^{L M}\left(s^{\prime}, s\right)\right)}=\left.\ln \sum_{i=1}^{N} e^{L_{i}}\right|_{D_{k}=+1}-\left.\ln \sum_{i=1}^{N} e^{L_{i}}\right|_{D_{k}=-1}= \\
= & \left.f\left(L_{1}, f\left(L_{2}, \ldots, f\left(L_{N-2}, f\left(L_{N-1}, L_{N}\right)\right)\right)\right)\right|_{D_{k}=+1}-\left.f\left(L_{1}, f\left(L_{2}, \ldots, f\left(L_{N-2}, f\left(L_{N-1}, L_{N}\right)\right)\right)\right)\right|_{D_{k}=-1}
\end{aligned}
$$

There is a need to complement this algorithm with using some approximation of the correction to improve the efficiency of the turbo decoder. The authors of this paper proposed an effective PL-log-MAP algorithm [13-16], which is not used complex functions (logarithm, exponential components sum) in the recursive process. This feature reduces the computational complexity. Next section gives some numerical results and simulation for PL-log-MAP algorithm.

\section{RESEARCH EXPERIMENTS FOR PL-LOG-MAP ALGORITHM}

In log-MAP algorithm at the stages of metrics and "soft" decisions calculation the important element is decreasing nonlinear function $f_{c o r}(z)$. It have been proposed to simplify calculations using standard linear functions in sub-ranges, which have been found via computer search by the least squares method [16]. So we take 6 sub-ranges: $z_{0} \in[0 ; 1)$, $z_{1} \in[1 ; 1.5), z_{2} \in[1.5 ; 2), z_{3} \in[2 ; 3), z_{4} \in[3 ; 4], z_{5} \in(4 ;+\infty)$. If $z$ is greater than 4 , the compensation term takes a small constant value, for example we set this measure on 0.01 .

So we see (Table 1), that our correction function is very precise on sub-ranges. The accuracy of our function is verified through the comparison against different approximations in table 2 for test range, where $M A E$ is Mean Absolute Error, $R M S E$ - Root-Mean-Square Error, $r$ is correlation coefficient, $\Delta$ - difference between $r$ for piecewise linear approximation and $r$ for other existing approximations in percents. Figure 1 shows the graphical comparisons for different correction function approximations.

The proposed function has high accuracy on the test range of the argument $z$ values (from 0 to 10 with step of 0.01 ) compared to existing analogs, such as fractional (by $0.11 \%$ ), quadratic (by $0.2 \%$ ), combined hybrid (by $0.37 \%$ ), Robertson lookup table (by $0.54 \%$ ), Zaytsev-linear (by $0.65 \%$ ), hybrid (by $0.69 \%$ ) and others [15-17].

Also we calculate the number of elementary mathematical operations (or computational complexity) for turbo decoding of one binary information symbol on digital signal processor [13]. The equation is presented in the next form

$$
f_{P L}(m, h) \approx 2^{m+2} \cdot h+33 \cdot 2^{m+1}-19,
$$

where $m$ is RSC coder memory; $h$ is total number of binary symbols from the output of the RSC coder.
TABle 1. Numerical Estimations for PL-LOG-MAP CORReCtion Term

\begin{tabular}{|l|c|c|c|c|c|}
\hline $\begin{array}{c}\text { Piecewise-linear } \\
\text { approximation }\end{array}$ & $\begin{array}{c}\text { Sub- } \\
\text { ranges }\end{array}$ & $\boldsymbol{a}_{i}$ & $\boldsymbol{b}_{\boldsymbol{i}}$ & $\boldsymbol{R M S E}$ & $\boldsymbol{r}_{\boldsymbol{i}}$ \\
\hline$f_{0}(z)=a_{0} \cdot z_{0}+b_{0}$ & {$[0 ; 1]$} & -0.3792 & 0.6754 & 0.0108 & 0.9968 \\
\hline$f_{1}(z)=a_{1} \cdot z_{1}+b_{1}$ & {$[1 ; 1,5]$} & -0.2229 & 0.5327 & 0.0019 & 0.9987 \\
\hline$f_{2}(z)=a_{2} \cdot z_{2}+b_{2}$ & {$[1,5 ; 2]$} & -0.1483 & 0.4213 & 0.0014 & 0.9984 \\
\hline$f_{3}(z)=a_{3} \cdot z_{3}+b_{3}$ & {$[2 ; 3]$} & -0.0773 & 0.2758 & 0.0032 & 0.9929 \\
\hline$f_{4}(z)=a_{4} \cdot z_{4}+b_{4}$ & {$[3 ; 4]$} & -0.0300 & 0.1362 & 0.0013 & 0.9922 \\
\hline$f_{5}(z)=a_{5} \cdot z_{5}+b_{5}$ & $(4 ; \infty)$ & -0.0000 & 0.0100 & - & - \\
\hline
\end{tabular}

TABle 2. Numerical Estimations fOR PL-LOG-MAP CORRection Term AGAINST OTHER CORRECTION TERMS

\begin{tabular}{|l|c|c|c|c|}
\hline \multicolumn{1}{|c|}{ Correction functions } & $\boldsymbol{M A E}$ & $\boldsymbol{R M S E}$ & $\boldsymbol{r}$ & $\Delta, \mathbf{\%}$ \\
\hline Proposed function & $\mathbf{0 . 0 0 5 9}$ & $\mathbf{0 . 0 0 7 0}$ & $\mathbf{0 . 9 9 9 4}$ & - \\
\hline S. Asoodeh & 0.0063 & 0.0089 & 0.9983 & 0.1100 \\
\hline L. Zhang, S.-Z. Yu & 0.0091 & 0.0135 & 0.9974 & 0.2000 \\
\hline S.V. Zaytsev & 0.0147 & 0.0172 & 0.9957 & 0.3700 \\
\hline P. Robertson et al , J. Wang & 0.0108 & 0.0170 & 0.9940 & 0.5400 \\
\hline S.V. Zaytsev & 0.0176 & 0.0243 & 0.9929 & 0.6500 \\
\hline L.L. Lim, D.W.G. Lim & 0.0227 & 0.0265 & 0.9925 & 0.6900 \\
\hline J.F. Cheng, T. Ottosson & 0.0179 & 0.0285 & 0.9860 & 1.3400 \\
\hline L.L. Lim, D.W.G. Lim & 0.0265 & 0.0453 & 0.9756 & 2.3800 \\
\hline S. Talakoub et al & 0.0342 & 0.0641 & 0.9351 & 6.4300 \\
\hline P. Gulak, W. Gross & 0.0432 & 0.0807 & 0.8941 & 10.5300 \\
\hline
\end{tabular}

For simulation the work of digital communication system we use the turbo codec [14] with two identical RSC codes with generator polynomial $(7,5)_{8}$, implemented in 1,4 and 8 iterations, with overall rate of $1 / 3$; random interleaver (S. Park-K. Miller random generator) with $m=2$ tail bits; AWGN channel for data modulated using BPSK; the energy per bit to noise power spectral density ratio $E_{b} / N_{0}=[0: 3]$ with step $0.25 d B ; 8$ decoding iterations; 1024 binary symbols in frame. Ziggurat algorithm was used for modeling standard normally distributed random variables. Stopping rule for turbo decoder is 10 frames with errors. The comparison with popular turbo decoding algorithms is presented in the Fig. 2 as a function BER $=f\left(E_{b} / N_{0}\right)$.

So the proposed PL-log-MAP algorithm almost achieves BER of log-MAP $(R M S E=0.0005, r=0.9998)$ and MAP (RMSE $=0.0004, r=0.9997)$ turbo decoding algorithms, but has a lower computational complexity. PL-log-MAP method works better than existing MAP modifications, especially in small $E_{b} / N_{0}$ area. If $E_{b} / N_{0}$ values is higher than $3-4 d B$ BER is not heavily dependent on the correction function. So BER of MAP modifications is approaching to BER of max-log-MAP. 

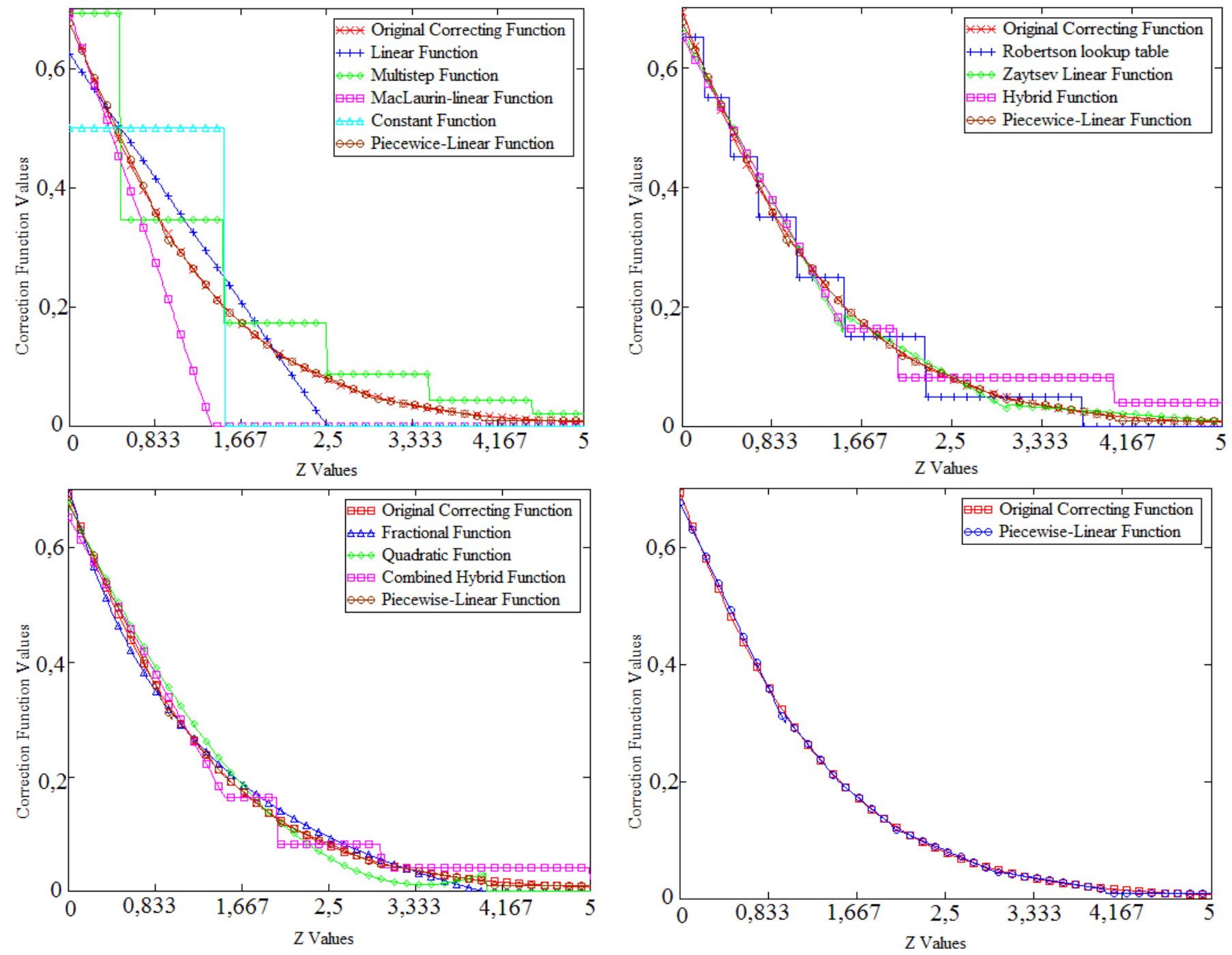

Fig. 1. GRAPHICAL COMPARISONS BETWEEN DIFFERENT CORRECTION FunCTIONS

Algorithms MAP, log-MAP, PL-log-MAP enable to get a significant energy gain compared to the SOVA $(0.5-0.6 d B$ for $\mathrm{BER}=10^{-3}-10^{-4}, 1.3 d B$ for BER $\left.=10^{-5}\right)$; Bi-SOVA, max-logMAP $(0.1-0.7 d B)$.

Also we performed another experiment with computer images. We worked with PL-log-MAP algorithm $(0-$ no coding, BPSK, 1, 4, 8 decoding iterations), $E_{b} / N_{0}=1 d B$ and the same settings as in the first experiment. During the information transmission process we used two computer images - "lena.jpg" (Lena Soderberg model, 768 frames, 4096 bits per frame) and "VNTU.jpg" (Vinnytsia National Technical University model, 681, 3632 bits per frame). The results of transmitted images during our experiment in MatLab are presented in Fig. 3(A) and 3(B). The BEN (bit errors number) and $S E N$ (symbol errors number) numerical results, as well as $B E R$ and $S E R$ (symbol error rate) performance are shown in table 3 for log-MAP and PL-log-MAP decoding algorithms for our experiment with images. Note, that we have used identical random interleavers for two decoding algorithms.

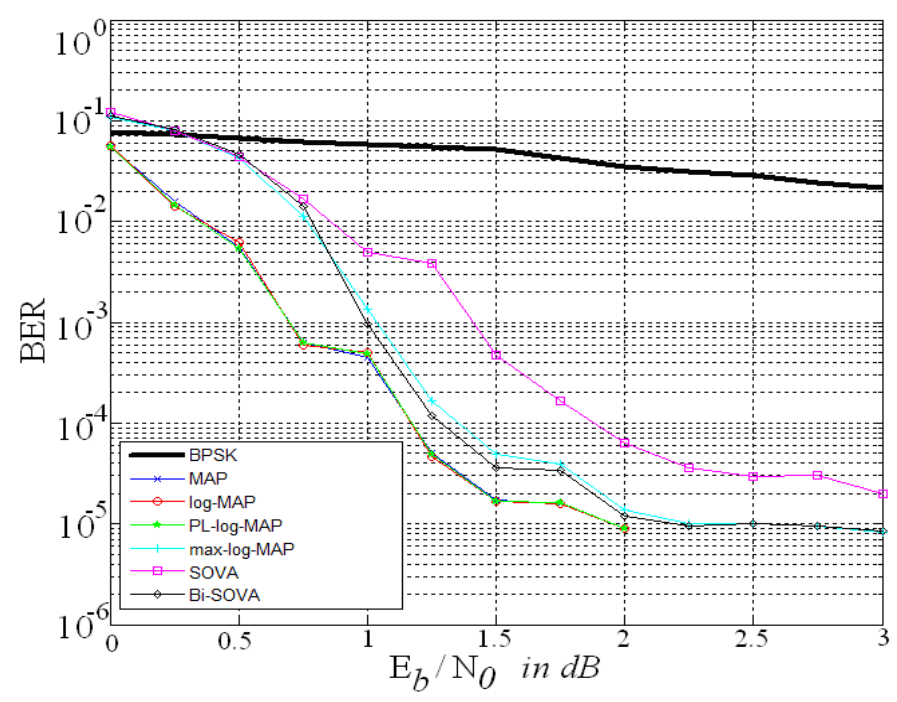

FIG. 2. EXPERIMENTAL ESTIMATIONS FOR BIT ERROR RATE 

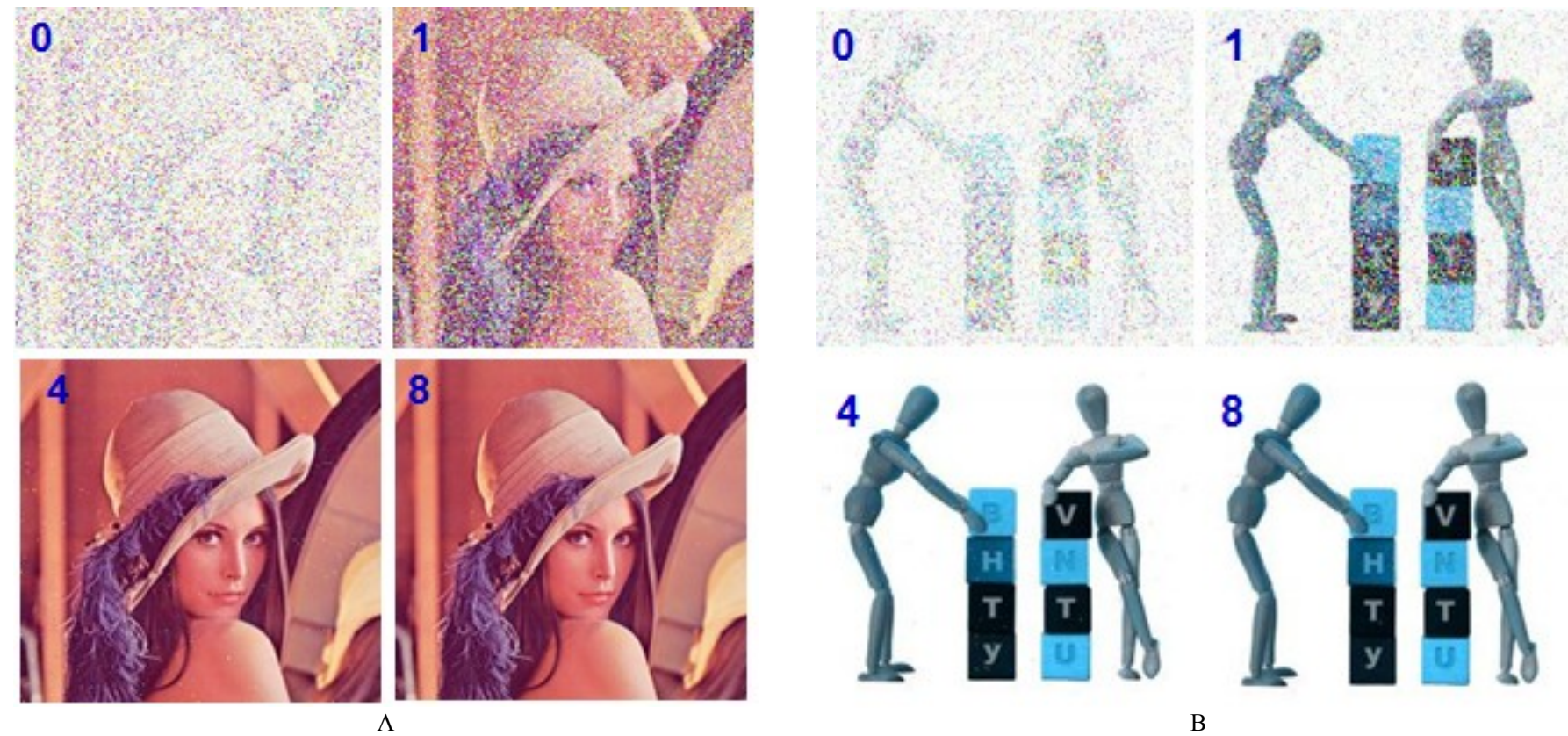

FIG. 3. TRANSMISSION PROCESS FOR EXPERIMENTAL IMAGES “LENA.JPG” (A) AND "VNTU.JPG” (B)

TABLE 3. BEN / SEN NuMERICAL RESUlts, BER / SER SimULATION FOR EXPERIMENTAL IMAGES "LENA.JPG" AND "VNTU.JPG"

\begin{tabular}{|c|c|c|c|c|c|}
\hline \multicolumn{6}{|c|}{ Experimental Image "lena.jpg" } \\
\hline \multirow{2}{*}{$\begin{array}{c}\mathbf{E}_{\mathbf{b}} / \mathbf{N}_{\mathbf{0}} \\
\mathbf{d B}\end{array}$} & \multirow{2}{*}{$\begin{array}{c}\text { Iterat. } \\
\text { № }\end{array}$} & \multicolumn{2}{|c|}{ log-MAP } & \multicolumn{2}{|c|}{ log-MAP } \\
\hline & & $B E N$ & SEN & BER & SER \\
\hline \multirow{4}{*}{$1 \mathrm{~dB}$} & 0 & 565822 & 188270 & 0.1799 & 0.9576 \\
\hline & 1 & 188419 & 107278 & 0.05989 & 0.5456 \\
\hline & 4 & 718 & 611 & $2.282 \cdot 10^{-4}$ & $3.108 \cdot 10^{-3}$ \\
\hline & 8 & 26 & 22 & $8.265 \cdot 10^{-6}$ & $1.119 \cdot 10^{-4}$ \\
\hline \multirow{2}{*}{$\begin{array}{c}\mathbf{E}_{\mathbf{b}} / \mathbf{N}_{\mathbf{0}} \\
\mathbf{d B}\end{array}$} & Iterat. & \multicolumn{2}{|c|}{ PL-log-MAP } & \multicolumn{2}{|c|}{ PL-log-MAP } \\
\hline & № & $B E N$ & SEN & BER & SER \\
\hline \multirow{3}{*}{$1 \mathrm{~dB}$} & 1 & 188419 & 107278 & 0.05989 & 0.5456 \\
\hline & 4 & 718 & 611 & $2.282 \cdot 10^{-4}$ & $3.108 \cdot 10^{-3}$ \\
\hline & 8 & 26 & 22 & $8.265 \cdot 10^{-6}$ & $1.119 \cdot 10^{-4}$ \\
\hline \multicolumn{6}{|c|}{ Experimental Image "VNTU.jpg" } \\
\hline \multirow{2}{*}{$\begin{array}{c}\mathbf{E}_{\mathbf{b}} / \mathbf{N}_{\mathbf{0}} \\
\mathbf{d B}\end{array}$} & Iterat. & \multicolumn{2}{|c|}{ log-MAP } & \multicolumn{2}{|c|}{ log-MAP } \\
\hline & № & $B E N$ & SEN & $B E R$ & $S E R$ \\
\hline \multirow{4}{*}{$1 \mathrm{~dB}$} & 0 & 501395 & 96535 & 0.1798 & 0.5537 \\
\hline & 1 & 165310 & 64551 & 0.05926 & 0.37027 \\
\hline & 4 & 606 & 359 & $2.173 \cdot 10^{-4}$ & $2.059 \cdot 10^{-3}$ \\
\hline & 8 & 13 & 4 & $4.661 \cdot 10^{-6}$ & $2.294 \cdot 10^{-5}$ \\
\hline \multirow{2}{*}{$\begin{array}{c}\mathbf{E}_{\mathbf{b}} / \mathbf{N}_{\mathbf{0}} \\
\mathbf{d B}\end{array}$} & Iterat. & \multicolumn{2}{|c|}{ PL-log-MAP } & \multicolumn{2}{|c|}{ PL-log-MAP } \\
\hline & № & $B E N$ & SEN & BER & SER \\
\hline \multirow{3}{*}{$1 \mathrm{~dB}$} & 1 & 165310 & 64551 & 0.05926 & 0.37027 \\
\hline & 4 & 606 & 359 & $2.173 \cdot 10^{-4}$ & $2.059 \cdot 10^{-3}$ \\
\hline & 8 & 13 & 4 & $4.661 \cdot 10^{-6}$ & $2.294 \cdot 10^{-5}$ \\
\hline
\end{tabular}

The results of these experiments confirm the high efficiency of PL-log-MAP turbo decoding algorithm. So it can be used in digital communication systems.

\section{CONCLUSIONS}

Thus, turbo code is widely used in different standards and commercial systems as highly efficient forward error correction method.

In this paper we have performed a brief overview of the most efficient turbo decoding algorithms, such as optimal
MAP and log-MAP as well as suboptimal simple PL-logMAP. The MAP algorithm is extremely complex. So have been developed a simplification of the MAP algorithm, which is called log-MAP. It offers the same optimal performance with a reasonable complexity. In PL-log-MAP algorithm we used the approximation of the correction term, that excludes computationally intensive operations with some deterioration of decoding quality.

To solve the problems of analysis, synthesis and operation of turbo codes in digital communication systems has been performed the simulation of data transmission with turbo codec. The researches showed, that BER characteristics is almost similar to those of MAP and log-MAP decoding algorithms for the same energy efficiency.

So simulation results confirm the high efficiency of PL-log-MAP turbo decoding algorithm. We conclude that it is suitable for using in digital communication systems.

\section{REFERENCES}

[1] C. Berrou, A. Glavieux, and P. Thitimajshima, "Near Shannon Limit Error-Correcting Coding and Decoding: Turbo-Codes," in ICC'93, Geneva, Switzerland, 1993, pp. 1064-1070.

[2] K. Gracie, M.-H. Hamon, "Turbo and Turbo-Like Codes: Principles and Applications in Telecommunications," Proceedings of the IEEE, vol. 95, 2007, pp. 1228-1254.

[3] C.E. Shannon, "A Mathematical Theory of Communication," Reprinted from The Bell System Technical Journal, vol 27, 1948, pp. 379-423, 623-656.

[4] J. Hagenauer, E. Offer, and L. Papke, "Iterative Decoding of Binary Block and Convolutional Codes," IEEE Transactions on Information Theory, № 2, vol. 42, 1996, pp. 429-445.

[5] J. Woodard, and L. Hanzo, "Comparative Study of Turbo Decoding Techniques: An Overview," IEEE Transactions on Vehicular Technology, №6, vol. 49, 2000, pp. 2208-2233.

[6] J. Hagenauer, P. Hoeher, "A Viterbi Algorithm with Soft-Decision Outputs and its Applications," Proc. in IEEE Global Telecommunications Conference, USA, Texas, 1989, pp. 1680-1686. 
[7] R. Morelos-Zaragoza, "The Art of Error Correction Coding," 2nd Edition, Chippenham, John Wiley \& Sons, 2006, 278 p.

[8] T.K. Moon, "Error Correcting Coding: Mathematical Methods and Algorithms," New Jersey, John Wiley \& Sons, 2005, 800 p.

[9] P. Robertson, E. Villebrun, and P. Hoeher, "A comparison of optimal and sub-optimal MAP decoding algorithms operating in the log domain," IEEE International Conference on Communications ICC'95 "Gateway to Globalization", Seattle, vol. 2, 1995, pp. 1009-1013.

[10] E. Boutillon, C. Douillard, and G. Montorsi, "Iterative Decoding of Concatenated Convolutional Codes: Implementation Issues," Proc. in IEEE, vol. 95, 2007, pp. 1201-1227.

[11] O.V. Stukach, A.N. Romanyuk, A.Ia. Kulyk, and Yu.Yu. Ivanov, "Modifications for Reduction Computational Complexity of the BCJR MAP Decoding Algorithm for Turbo Coding Constructions," Proceedings of DonNTU. Informatics, Cybernetics and Computer Science, Krasnoarmiysk, vol. 1, 2015, pp. 107-112. (in Russian).

[12] L. Bahl, J. Cocke, F. Jelinek, and J. Raviv, "Optimal Decoding of Linear Codes for Minimizing Symbol Error Rate," IEEE Transactions on Information Theory, vol. 20, 1974, pp. 284-287.

[13] Yu.Yu. Ivanov, "Hardware and Software Features of the Turbo Codes: Analysis of the Implementation Complexity on Digital Signal Processor," Journal of Vinnytsia Polytechnical Institute, Vinnytsia, vol. 3, 2016, pp. 94-101. (in Ukrainian)

[14] Yu.Yu. Ivanov, "Experimental Research of Turbo Codes Error-Correction Characteristics: Numerical Estimations and Simulation for Novel Suboptimal PL-Log-MAP Decoding Algorithm," Journal of Vinnytsia Polytechnical Institute, Vinnytsia, vol. 5, 2016, pp. 76-84. (in Ukrainian)

[15] Yu.Yu. Ivanov, A.N. Romanyuk, A.Ia. Kulyk, and O.V. Stukach, " $A$ Novel Suboptimal Piecewise-Linear-log-MAP Algorithm for Turbo Decoding," Proc. on XI IEEE International Siberian Conference on Control and Communications, 2015, pp. 1-8, DOI: 10.1109/SIBCON.2015.7147195.

[16] Yu.Yu. Ivanov, "Error-correction Decoding of Turbo Codes in Distributed Computer Systems," Ph.D. dissertation, Vinnytsia National Technical University, Ukraine, 2016, 174 p. (in Ukrainian)

[17] S.V. Zaytsev, "The Modified log-MAP Decoding Algorithm for Turbo Codes Taking into Account the Impact of Intentional Interference," Mathematical Machines and Systems, № 4, 2015, pp. 70-79 (in Ukrainian). 